Original Article

Joumal of Epilepsy Research pISSN 2233-6249 / eISSN 2233-6257

Received December 1, 2020

Revised December 15, 2020

Accepted December 22, 2020

Corresponding author:

Richard A. Prayson, MD, MEd

Robert J. Tomsich Pathology \& Laboratory

Medicine Institute, Cleveland Clinic, 9500

Euclid Ave, Cleveland, OH 44195, USA

Tel. +1-216-444-8805

Fax. +1-216-445-6967

E-mail; praysor@ccf.org

\title{
Molecular Immunohistochemical Profile of Angiocentric Glioma
}

\author{
Lanisha D. Fuller, MD, Richard A. Prayson, MD, MEd \\ Robert J. Tomsich Pathology \& Laboratory Medicine Institute, Cleveland Clinic, Cleveland, OH, USA
}

Background and Purpose: Angiocentric glioma is a rare, World Health Organization grade I tumor that is seen predominantly in children and young adults and typically presents with seizures. Histologically, it shows features of both infiltrating glioma and ependymoma.

Methods: We examined molecular immunohistochemical markers which could help in distinguishing this entity from its differential diagnostic considerations.

Results: We retrospectively reviewed the clinicopathologic features of angiocentric gliomas and performed immunohistochemical staining for isocitrate dehydrogenase 1 (IDH-1) (R132H), p53, ATRX, BRAF V600E, $\mathrm{Ki}-67$, and H3 K27M on formalin-fixed, paraffin-embedded tissue. Seven cases in total were found and included six excisional specimens and one biopsy. ATRX staining was retained in all cases. There was no evidence of staining with antibodies to IDH-1 (R132H), H3 K27M, or BRAF V600E. Five tumors showed no staining with antibody to $\mathrm{p} 53$ and two tumors showed less than $5 \%$ positivity. Ki- 67 indices were less than $1 \%$ in five tumors, $4-5 \%$ in one tumor, and $9-10 \%$ in one tumor.

Conclusions: In conclusion, the immunohistochemical markers for ATRX, p53, IDH-1 (R132H), BRAF V600E, H3 K27M show wild-type staining, potentially aiding in avoiding misdiagnoses in cases morphologically similar to other low-grade gliomas. Ki-67 labeling indices are low in most tumors. (2020;10: 79-83)

Key words: Angiocentric glioma, IDH-1, ATRX, BRAF, H3 K27M, p53

\section{Introduction}

Angiocentric glioma was first officially recognized as a distinct entity in the 2007 edition of the World Health Organization (WHO) Classification of Tumours of the Central Nervous System. 'It is a rare, grade I tumor that is seen predominantly in children and young adults without gender predilection. ${ }^{2}$ Clinically, patients most commonly present with pharmacoresistant seizures. ${ }^{2}$ Angiocentric gliomas usually have a good prognosis following gross total resection and the rate of tumor recurrence is low. ${ }^{2}$ Histologically and ultrastructurally, they share features with astrocytomas and ependymomas. ${ }^{3}$ They are characterized by infiltrating, monomorphous, bipolar tumor cells that are marked by an angiocentric growth pattern.

To our knowledge, few studies have examined the immunohistochemical molecular markers p53, ATRX, isocitrate dehydrogenase 1 (IDH-1) (R132H), H3K27M, and BRAF V600E in angiocentric gliomas. Such markers may potentially prove useful in distinguishing this tumor from other tumors in its morphologic differ- ential diagnosis when molecular methods are not available. This study retrospectively evaluates a series of immunohistochemical molecular markers in seven angiocentric gliomas.

\section{Methods}

With institutional review board approval, a retrospective search of the pathology database was conducted for "angiocentric glioma". Seven tumors were identified between the years 1999 to 2020 and comprised the study group. Cases were reviewed and diagnoses corroborated according to the 2016 WHO Classification of Tumours of the Central Nervous System. ${ }^{4}$ Clinical data was obtained from the electronic medical records and included patient age at diagnosis, gender, clinical presentation, location of tumor, and outcome. Hematoxylin and eosin-stained slides were reviewed to confirm the diagnoses in these cases. Immunohistochemical staining for IDH-1 (R132H) (1:40 dilution; Dianova, Hamburg, Germany), p53 (1:20 dilution; Dako, Santa Clara, CA, USA), alpha-thalassemia X-linked 
mental retardation syndrome (ATRX) (1:200 dilution; Sigma-Aldrich, St. Louis, MO, USA), BRAF V600E (1:175 dilution; Spring Bioscience, Pleasanton, CA, USA), Ki-67 (prediluted; Ventana, Oro Valley, AZ, USA), and H3 K27M (1:500 dilution; Abcam, Cambridge, UK) was performed on formalin-fixed, paraffin-embedded tissue. Ki-67 labeling indices were determined by assessing the percentage of positive staining nuclei in 1,000 tumor cells in the area of tumor with the most staining. The three-tiered International League Against Epilepsy (ILAE) classification system for focal cortical dysplasias ${ }^{5}$ was used to classify cases in which adjacent focal cortical dysplasia was present. Using the same classification system, we additionally classified the cortical dysplasias as if tumor was absent (i.e., as resembling a type I or Il pattern), in order to better describe the histology.

\section{Results}

Clinical features are outlined for each patient in Table 1. A total of seven cases, five males and two females, with a median age of 15 years (range, 6-56) at diagnosis, were identified and comprised the study group. The cases included six excisional specimens and one biopsy. On imaging, all tumors had the appearance of a low grade infiltrative glioma (Fig. 1).

Histologically, all tumors showed perivascular pseudorosette formations (Fig. 2A). An infiltrative border was discernible in six of six cases with sufficient tissue for evaluation. Calcifications were observed in two of seven cases. Mitotic activity, necrosis and vascular proliferation were not observed. Five of six cases with sufficient tissue for evaluation showed adjacent focal cortical dysplasia (ILAE type IIIb). In the absence of tumor, the patterns of dysplasia seen in all cases resembled type Ib lesions, which histologically translates into abnormal tangential cortical lamination (Fig. 2B).

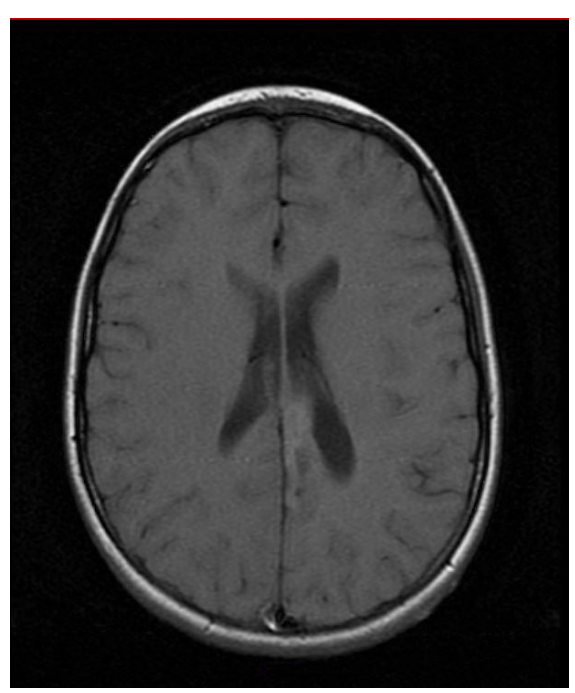

Figure 1. Representative T1-weighted, axial magnetic resonance image of angiocentric glioma (patient 4). Imaging revealed a cortical and subcortical lesion in the inferior left parietal lobe and anterior cingulate gyrus.

Table 1. Summary of clinicopathologic features of angiocentric gliomas

\begin{tabular}{|c|c|c|c|c|c|c|c|}
\hline Patient & $\begin{array}{l}\text { Age at } \\
\text { diagnosis } \\
\text { (years) }\end{array}$ & Gender & $\begin{array}{l}\text { Symptoms at } \\
\text { presentation }\end{array}$ & Tumor location & FCD & $\begin{array}{l}\text { Follow-up } \\
\text { length } \\
\text { (months) }\end{array}$ & Status at last follow-up \\
\hline 1 & 10 & $\mathrm{~F}$ & Seizures & Left parietal lobe & Yes & 237 & Seizure free, no other symptoms \\
\hline 2 & 20 & M & Seizures & Left parietal lobe & Yes & 148 & Seizures \\
\hline 3 & 15 & M & Headache & $\begin{array}{l}\text { Ventricular wall and } \\
\text { thalamic region }\end{array}$ & NA & 125 & Stable tumor; headaches \\
\hline 4 & 15 & $\mathrm{~F}$ & Seizures & $\begin{array}{l}\text { Inferior left parietal } \\
\text { lobe and anterior } \\
\text { cingulate gyrus }\end{array}$ & Yes & 98 & Seizures \\
\hline 5 & 6 & M & $\begin{array}{l}\text { Intermittent nausea } \\
\text { and vomiting }\end{array}$ & $\begin{array}{l}\text { Right anterior } \\
\text { temporal lobe }\end{array}$ & Yes & 51 & Unclear \\
\hline 6 & 56 & M & $\begin{array}{l}\text { Aphasia, confusion, } \\
\text { difficulty following } \\
\text { commands }\end{array}$ & Left temporal lobe & No & 3 & $\begin{array}{l}\text { Persistent speech difficulty and } \\
\text { intermittent headache }\end{array}$ \\
\hline 7 & 15 & M & Seizures, headache & Left temporal lobe & Yes & 224 & $\begin{array}{l}\text { Seizure free on medication, residual } \\
\text { speech impairment, gait } \\
\text { impairment and headaches, right } \\
\text { sensory loss and peripheral vision } \\
\text { loss }\end{array}$ \\
\hline
\end{tabular}

FCD, focal cortical dysplasia; $F$, female; $M$, male; NA, not assessed. 
Immunohistochemical stains were performed in all cases when possible. For case 3, only Ki-67 was performed due to scant amount of tissue. Immunohistochemistry revealed that ATRX staining was retained in all examined cases ( $n=6$ ) (Fig. 2C). There was no evidence of staining with antibodies to IDH-1 (R132H) (n=6), H3 K27M ( $n=6)$, or BRAF V600E $(n=6)$. Five tumors showed no staining with antibody to $\mathrm{p} 53$ and two tumors showed less than 5\% positivity (Fig. 2D). Ki-67 indices were less than $1 \%$ in five tumors (Fig. 2E), 4-5\% in one tumor, and $9-10 \%$ in one tumor.

\section{Discussion}

In 2015, Ampie et al. ${ }^{2}$ published a literature review on angiocentric gliomas which included a total of 88 patients (four of the 88 patients are part of our current study and were previously published by Marburger and Prayson ${ }^{3}$ ). Ampie and colleagues ${ }^{2}$ found that the average age at diagnosis was 16 years and the most common symptom at presentation was seizures (86\%). In keeping with previously reported clinical presentations, the majority of our patients were children or young adults initially presenting with seizures. All lesions except one involved either the parietal or temporal lobe. All patients were alive at the last known date of follow-up. While none of the gross totally resected tumors recurred, outcomes differed between the cases with only two patients confirmed to have no residual symptoms without medication.

Most cases with sufficient tissue for evaluation had adjacent focal cortical dysplasia, similar to other low grade pharmacoresistant epilepsy-associated tumors (e.g., ganglioglioma, dysembryoplastic neuroepithelial tumor). ${ }^{6}$ In order to describe the histology of the cortical dysplasias better, we classified each lesion as if tumor was absent. Using this method, all our cases with focal cortical dysplasia had patterns resembling type $\mathrm{lb}$ lesions

With the 2016 WHO edition and in recent years, several immunohistochemical diagnostic and prognostic markers have been added to the diagnostic criteria of gliomas. So far, the staining pattern for those newer markers has not been described for every glioma entity. We investigated several of those markers potentially useful in differentiating other gliomas from angiocentric glioma. Among differential diagnostic considerations that differ in prognosis/outcome and treatment are diffuse astrocytoma, pilocytic astrocytoma and diffuse midline glioma.

$70-80 \%$ of low-grade diffuse gliomas have mutations in IDH- $1 .^{7}$ IDH-1 (R132H) represents the vast majority of IDH mutations (90\%). ${ }^{8}$ An IDH mutation per definition is always present in oligodendrogliomas and is often present in diffuse astrocytom as (the existence of diffuse astrocytoma without an IDH mutation is currently
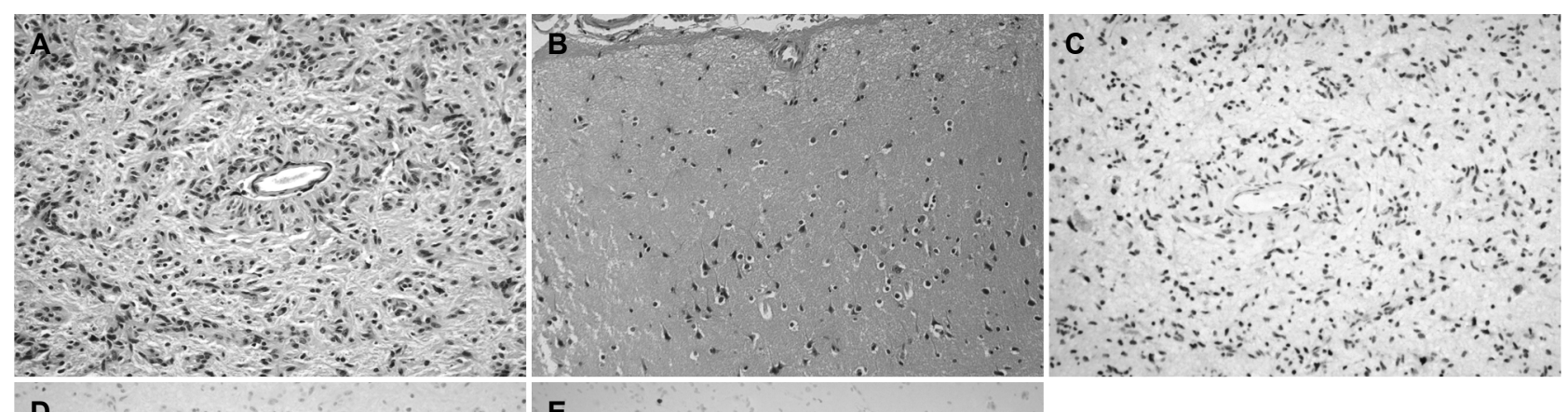

D

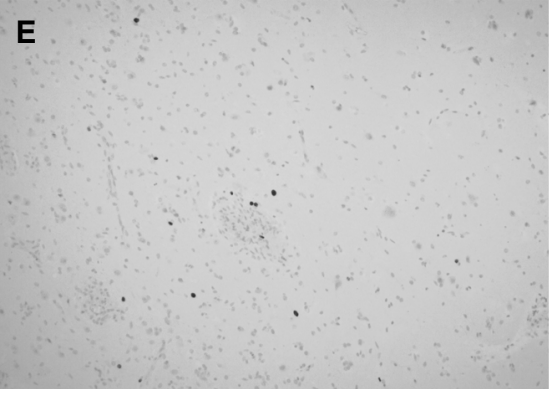

Figure 2. Angiocentric glioma. (A) The tumor is composed of cytologically bland, bipolar cells forming perivascular pseudorosettes. (B) ILAE type Ib FCD was noted adjacent to the tumor, marked by cortical architectural disorganization with large pyramidal neurons located where cortical layer 2 typically resides. (C) Representative case of angiocentric glioma (patient 5) showing retained ATRX. (D) This case also showed less than 5\% positivity for p53. (E) the Ki-67 index was less than $1 \%$ (A, B: hematoxylin and eosin, original magnification, $\times 200$; C, D: original magnification, $\times 200$; E: original magnifications, $\times 100$ ). 
being debated in the literature ${ }^{9}$ ). Diffuse midline gliomas seem to not have IDH mutations as Solomon et al. ${ }^{10}$ showed in their 47-case study. IDH mutations have only been reported infrequently in pilocytic astrocytomas. ${ }^{11}$ We found that angiocentric gliomas show no staining for IDH-1 (R132H), inferring that positive staining would exclude a diagnosis of angiocentric glioma. This is consistent with findings of previous studies that, combined, stained thirteen cases of angiocentric glioma with IDH-1 (R132H) antibody and all cases were negative. ${ }^{12-14}$

ATRX and/or TP53 mutations in diffuse glioma are considered consistent with an astrocytic genotype. ${ }^{15,16}$ About $90 \%$ of IDH mutant low-grade gliomas without chromosomes $1 p$ and $19 q$ codeletion show p53 and ATRX mutations. ${ }^{16,17}$ ATRX is usually retained in pilocytic astrocytomas. ${ }^{16}$

A study of 61 diffuse midline gliomas found p53 to be positive in 40 of their cases (66\%) and they observed ATRX loss in 17 cases $(28 \%) .{ }^{18}$ We found that angiocentric gliomas retain staining for ATRX and have no or insignificant p53 staining. This means that in a case with loss of ATRX and diffuse staining for p53, an angiocentric glioma could most likely be excluded. Retained staining for ATRX and absent or low p53 staining does not point to either diagnosis.

BRAF V600E mutations occur in 5-10\% of pilocytic astrocytomas and $50 \%$ of gangliogliomas. ${ }^{19}$ Chi et al. ${ }^{20}$ reviewed the literature and found only $2 / 251(0.8 \%)$ diffuse astrocytomas to be positive for BRAF V600 mutations. None of our angiocentric gliomas stained for BRAF V600E. Hence, a positive staining case is less likely to be an angiocentric glioma.

The majority of angiocentric gliomas are located in the temporal, frontal, or parietal lobe, however, they have also been reported in midline structures. ${ }^{2} \mathrm{~A}$ glioma that arises in the midline raises the possibility of a diffuse midline glioma, H3 K27M-mutant. Other gliomas in the differential of angiocentric glioma, like pilocytic astrocytoma, have reported mutant cases. ${ }^{15}$ Chan et al. ${ }^{14}$ stained one angiocentric glioma located in a midline structure for H3 K27M and reported negative staining. All our cases were negative, including one case located in the ventricular wall and thalamic region.

Angiocentric gliomas are known to have a low mitotic count and the Ki-67 proliferative indices range from less than $1 \%$ to $12 \%$ in the literature, with most cases showing indices of less than $1 \%$. ${ }^{2,3,12}$ Our Ki-67 staining was consistent with the generally low proliferative indices previously reported.

Due to the rarity of this entity, a limiting factor of our study is the small sample size. Even when taking into account the information gathered from published cases, the total number of cases is small. Additionally, not every published case has evaluated the same immunohistochemical markers, essentially further reducing the cohort size.

In conclusion, many molecular immunohistochemical markers that are frequently encountered in other low grade tumors, such as diffuse astrocytoma (ATRX loss, p53 positive), low grade oligodendroglioma and diffuse astrocytoma (IDH1 (R132H) mutated), pilocytic astrocytoma and ganglioglioma (BRAF V600E mutated), and diffuse midline glioma (H3 K27M mutated), seem to not be present in angiocentric glioma; these markers can be potentially employed in avoiding misdiagnosis.

\section{Conflict of Interest}

The authors declare that they have no conflicts of interest.

\section{References}

1. Louis DN, Ohgaki H, Wiestler OD, Cavenee WK. WHO Classification of Tumours of the Central Nervous System. 4th ed. Lyon: IARC Press, 2007.

2. Ampie L, Choy W, DiDomenico JD, et al. Clinical attributes and surgical outcomes of angiocentric gliomas. J Clin Neurosci 2016;28:117-22.

3. Marburger T, Prayson R. Angiocentric glioma: a clinicopathologic review of 5 tumors with identification of associated cortical dysplasia. Arch Pathol Lab Med 2011;135:1037-41.

4. Louis DN, Ohgaki H, Wiestler OD, et al. WHO Classification of Tumours of the Central Nervous System. 4th ed. Lyon: International Agency for Research on Cancer, 2016;119-20.

5. Blümcke I, Thom M, Aronica $E$, et al. The clinicopathologic spectrum of focal cortical dysplasias: a consensus classification proposed by an ad hoc TASK FORCE of the ILAE Diagnostic Methods Commission. Epilepsia 2011;52:158-74.

6. Frater JL, Prayson RA, Morris III HH, Bingaman WE. Surgical pathologic findings of extratemporal-based intractable epilepsy: a study of 133 consecutive resections. Arch Pathol Lab Med 2000;124:545-9.

7. Kim YH, Nobusawa S, Mittelbronn M, et al. Molecular classification of low-grade diffuse gliomas. Am J Pathol 2010;177:2708-14.

8. Lee SC. Diffuse gliomas for nonneuropathologists: the new integrated molecular diagnostics. Arch Pathol Lab Med 2018;142:804-14.

9. Brat DJ, Aldape K, Colman H, et al. cIMPACT-NOW update 3: recommended diagnostic criteria for "diffuse astrocytic glioma, IDH-wildtype, with molecular features of glioblastoma, WHO grade IV". Acta Neuropathol 2018;136:805-10.

10. Solomon DA, Wood MD, Tihan $T$, et al. Diffuse midline gliomas with histone H3-K27M mutation: a series of 47 cases assessing the spectrum of morphologic variation and associated genetic alterations. Brain Pathol 
2016:26:569-80.

11. Prayson RA. IDH-1 polymorphisms in pilocytic astrocytomas. Ann Diagn Pathol 2019;43:151411.

12. Ni HC, Chen SY, Chen L, Lu DH, Fu YJ, Piao YS. Angiocentric glioma: a report of nine new cases, including four with atypical histological features. Neuropathol Appl Neurobiol 2015;41:333-46.

13. Raghunathan A, Olar A, Vogel $H$, et al. Isocitrate dehydrogenase 1 R132H mutation is not detected in angiocentric glioma. Ann Diagn Pathol 2012;16:255-9.

14. Chan E, Bollen AW, Sirohi D, et al. Angiocentric glioma with MYB-QKI fusion located in the brainstem, rather than cerebral cortex. Acta Neuropathol 2017;134:671-3.

15. Louis DN, Giannini C, Capper D, et al. cIMPACT-NOW update 2: diagnostic clarifications for diffuse midline glioma, H3 K27M-mutant and diffuse astrocytoma/anaplastic astrocytoma, IDH-mutant. Acta Neuropathol
2018;135:639-42.

16. Ebrahimi A, Skardelly $M$, Bonzheim I, et al. ATRX immunostaining predicts IDH and H3F3A status in gliomas. Acta Neuropathol Commun 2016;4:60.

17. Cancer Genome Atlas Research Network, Brat DJ, Verhaak RG, et al. Comprehensive, integrative genomic analysis of diffuse lower-grade gliomas. N Eng/ J Med 2015;372:2481-98.

18. Wang L, Li Z, Zhang M, et al. H3 K27M-mutant diffuse midline gliomas in different anatomical locations. Hum Pathol 2018;78:89-96.

19. Behling F, Schittenhelm J. Oncogenic BRAF alterations and their role in brain tumors. Cancers (Basel) 2019;11:794.

20. Chi AS, Batchelor TT, Yang D, et al. BRAF V600E mutation identifies a subset of low-grade diffusely infiltrating gliomas in adults. J Clin Oncol 2013;31:e233-6. 